\title{
MEG evoked responses and rhythmic activity provide spatiotemporally complementary measures of neural activity in language production
}

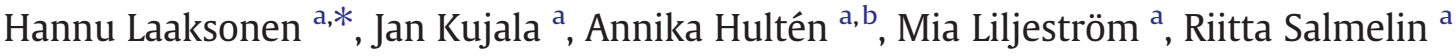 \\ a Brain Research Unit, Low Temperature Laboratory, Aalto University, Finland \\ b Department of Psychology, Åbo Akademi University, Finland
}

\section{A R T I C L E I N F O}

Article history:

Received 14 October 2011

Revised 27 November 2011

Accepted 28 November 2011

Available online 7 December 2011

\section{Keywords:}

MEG

Cortical oscillatory activity

Evoked response

Beamforming

Picture naming

\begin{abstract}
A B S T R A C T
Phase-locked evoked responses and event-related modulations of spontaneous rhythmic activity are the two main approaches used to quantify stimulus- or task-related changes in electrophysiological measures. The relationship between the two has been widely theorized upon but empirical research has been limited to the primary visual and sensorimotor cortex. However, both evoked responses and rhythms have been used as markers of neural activity in paradigms ranging from simple sensory to complex cognitive tasks. While some spatial agreement between the two phenomena has been observed, typically only one of the measures has been used in any given study, thus disallowing a direct evaluation of their exact spatiotemporal relationship. In this study, we sought to systematically clarify the connection between evoked responses and rhythmic activity. Using both measures, we identified the spatiotemporal patterns of task effects in three magnetoencephalography (MEG) data sets, all variants of a picture naming task. Evoked responses and rhythmic modulation yielded largely separate networks, with spatial overlap mainly in the sensorimotor and primary visual areas. Moreover, in the cortical regions that were identified with both measures the experimental effects they conveyed differed in terms of timing and function. Our results suggest that the two phenomena are largely detached and that both measures are needed for an accurate portrayal of brain activity.
\end{abstract}

(c) 2011 Elsevier Inc. All rights reserved.

\section{Introduction}

In recordings of brain electrophysiology, at the neural population level, the amount of activation has most often been estimated using two different measures. On the one hand, events locked to stimulus or task timing have been averaged to obtain so-called evoked responses. On the other hand, the cerebral cortex shows rhythmic activity, which has been investigated both in its spontaneous manifestation and as responses induced by stimuli or tasks (Hari and Salmelin, 1997).

Spontaneous rhythmic activity as measured by noninvasive imaging methods, at rest, is detected consistently in and around the primary visual cortex and the parieto-occipital sulcus, mostly at $\sim 10 \mathrm{~Hz}$ ("alpha"), and in and around the hand representation area in the central sulcus, with both $10-\mathrm{Hz}$ and $20-\mathrm{Hz}$ ("beta") components (together, "mu" rhythm) (Hari and Salmelin, 1997). Sensory stimulation or tasks tend to modulate the cortical rhythms prevalent at the respective sensory area. The modulations are not limited to sensory events only; e.g., an increased level of the parieto-occipital $10-\mathrm{Hz}$ rhythm has been linked to memory load (Jensen et al., 2002; Tuladhar et al., 2007) and modulation of the motor cortical $20-\mathrm{Hz}$ rhythm has been observed in language production tasks, also reflecting cognitive aspects (Saarinen et al., 2006;

\footnotetext{
* Corresponding author at: Brain Research Unit, Low Temperature Laboratory, Aalto University, P.O. Box 15100, FI-00076 AALTO, Finland.

E-mail address: hannu@neuro.hut.fi (H. Laaksonen).
}

Salmelin et al., 2000). In some cognitive tasks, reactivity of rhythmic activity has additionally been reported at $\sim 6-8 \mathrm{~Hz}$ ("theta") in the frontal cortex (Ishii et al., 1999; Jensen and Tesche, 2002; Onton et al., 2005). Intracranial electroencephalography (EEG) recordings have emphasized the role of the $~ 30-100-\mathrm{Hz}$ range ("gamma") in task performance, particularly frequencies above $60 \mathrm{~Hz}$ ("high gamma") (Jerbi et al., 2009). However, with the non-invasive MEG recordings, gamma has been observed essentially in the visual cortex (Hoogenboom et al., 2006; Muthukumaraswamy et al., 2010; Tallon-Baudry et al., 1997).

Phase-locked evoked responses are typically evident within about $1 \mathrm{~s}$ from a selected trigger point. In sensory and motor stimulation and task performance, their cortical sources are concentrated to the sensory and motor representation areas, whereas in higher-order cognitive tasks they can be found in many diverse cortical areas, illustrating that evoked responses may be present throughout the cortex (Brenner et al., 1975; Brenner et al., 1978; Hari et al., 1980; Helenius et al., 1998; Salmelin, 2007; Salmelin et al., 1994). In this study we considered the cognitive task of picture naming that is thought to incorporate all major aspects of word production and progress from visual analysis and recognition through activation of meaning and sound form to articulation. As described by evoked responses, the cortical sequence of activation in picture naming starts with an early, transient occipital response $(<200 \mathrm{~ms})$ and proceeds with more sustained responses in the parietal and temporal areas ( $>200 \mathrm{~ms}$ ) and often, in the frontal cortex (>300 ms) (Hultén et al., 
2009; Indefrey and Levelt, 2004; Liljeström et al., 2009; Salmelin, 2007; Salmelin et al., 1994; Vihla et al., 2006).

The exact spatiotemporal relationship between evoked responses and modulation of cortical rhythms continues to be under debate. According to the so-called additive model, evoked responses are generated by transient brain events which do not affect the ongoing oscillatory activity (Mäkinen et al., 2005; Mazaheri and Jensen, 2006; Shah et al., 2004). However, in a few cases, partial phase resetting of ongoing oscillations has been shown to give rise to evoked responses (Makeig et al., 2002; Penny et al., 2002). Evoked responses have also been hypothesized to arise from an asymmetry of rhythmic amplitudes, i.e., the oscillations do not have a zero mean but, rather, the peaks are modulated more than the troughs (Mazaheri and Jensen, 2008; Nikulin et al., 2007). This asymmetry forms a slow evoked response when averaged over multiple repetitions. When similar paradigms, particularly those focusing on low-level visual or somatosensory processing, have been used to study either evoked responses or modulation of rhythmic activity, the two types of phenomena have been found to be generated in essentially the same areas in the occipital cortex and around the parieto-occipital sulcus (visual), and along the central sulcus (somatosensory) (Hari and Salmelin, 1997; Karhu et al., 1991; Vanni et al., 1996; Vanni et al., 1997). Yet, when evoked responses and modulation of rhythmic activity have been analyzed from the same data set, differences have been observed in task-related functionality and hemispheric balance (e.g., Salmelin et al., 2000; Schnitzler et al., 1997). Correspondence between the two measures in other, presumably functionally higherlevel areas has not been extensively looked into. In general, modulations of oscillatory power last longer than transient evoked responses, with the protracted duration suggested to be beneficial for combining information from sequential events (Dinse et al., 1997). Most often, however, MEG and EEG studies have focused on only one of these measures.

Here, we sought to clarify the connection between rhythmic activity and evoked responses by analyzing MEG data in high-level cognitive processing. The full data set included three experiments, with altogether 31 individual recordings, that all addressed the sequence from visual perception to language production, using a picture naming paradigm (Hultén et al., 2009; Liljeström et al., 2009; Vihla et al., 2006). Our aim was to identify the activation patterns (source areas, time courses) one would typically find when focusing on either evoked responses or rhythmic brain activity.

As these two markers of neural activity are inherently different in nature, the solutions for bridging the gap from sensor-level to sourcelevel descriptions also tend to be somewhat different. We start the evaluation with a model-free comparison of sensor-level distributions of evoked responses and modulations of rhythmic activity. However, this approach necessarily provides only a relatively cursory view of the spatial relationships. Therefore, we then reach to the source level, aiming for maximal sensitivity and specificity attainable with each measure by using well-established and reliable methods that are optimally suited for their specific modeling purposes. Equivalent current dipole modeling (ECD) of evoked responses was available for all three paradigms (Hultén et al., 2009; Liljeström et al., 2009; Vihla et al., 2006), also supported by minimum norm estimates (MNE) analysis (Liljeström et al., 2009). For spatiotemporal mapping of rhythmic activity, frequency-domain event-related DICS (Dynamic Imaging of Coherent Sources, DICS; Gross et al., 2001; erDICS, Laaksonen et al., 2008) was here applied on these same three data sets. erDICS is an accurate and versatile tool for identifying timevariant modulations of oscillatory activity (Laaksonen et al., 2008). DICS/erDICS is better suited for analysis of rhythmic activity than the ECD or MNE (or MCE, minimum current estimate) methods that are commonly used for analysis of evoked responses (Liljeström et al., 2005). The primary goal in the present study was to determine, on an individual level, areas that showed event-related modulation of rhythmic activity (suppression/enhancement, often referred to as event-related de/synchronization; Pfurtscheller and Aranibar, 1977), and to compare that spatial description with the neural sources of the phase-locked evoked responses.

We investigated the $1-90 \mathrm{~Hz}$ range which covers the typical frequencies of interest in EEG/MEG studies, with specific emphasis on the most prominent frequency bands in the MEG spectrum at $\sim 10 \mathrm{~Hz}$ and $\sim 20 \mathrm{~Hz}$. Individual-level identification of both evoked responses and rhythmic activity, in a large number of subjects, allowed a robust, direct evaluation of the spatiotemporal correspondence between the two measures of neural activation. In addition, when both evoked responses and modulation of rhythmic activity were detected in the same brain area, their time courses and task dependence could be directly compared.

\section{Methods}

\section{MEG recording and preprocessing}

The MEG data were measured with a Neuromag Vectorview ${ }^{\mathrm{TM}}$ 306-channel neuromagnetometer (Elekta Oy, Helsinki, Finland). The measurement device contains 102 sensor elements arranged in a helmet-shaped array, with two orthogonal planar gradiometers and one magnetometer per element. The planar gradiometers were used in this study, as they provide more accurate spatial information already at the sensor level and are not as sensitive to external noise as magnetometers (Hämäläinen et al., 1993). The data was bandpass filtered to $0.03-200 \mathrm{~Hz}$ and sampled at $600 \mathrm{~Hz}$. All data were preprocessed with MaxFilter (software by Elekta Oy), using the temporal extension of the signal space separation (tSSS, Taulu et al., 2004) method with buffer length of $16 \mathrm{~s}$ and correlation limit of 0.9 . All data sets were transformed into the same head position with MaxFilter.

We used data from three separate experiments, all with variants of overt picture naming and silent processing of the item name (covert picture naming or categorization by item name): object naming/categorization (Vihla et al., 2006), overt/covert object naming (Liljeström et al., 2009) and naming/categorization of newly learned objects (Hultén et al., 2009). The experimental designs are illustrated in Fig. 1. Altogether 31 individual data sets were available across the studies, with 10-11 participants per experiment; a few individuals participated in more than one study.

\section{Object naming/categorization}

Ten healthy subjects ( 5 females, 5 males) were shown black-andwhite drawings of common objects (60 images) (Fig. 1A). Each stimulus was displayed for $150 \mathrm{~ms}$, followed by a blank screen for $850 \mathrm{~ms}$. A question mark was then presented for $1 \mathrm{~s}$, prompting the subject to respond. The present study focused on two of the original

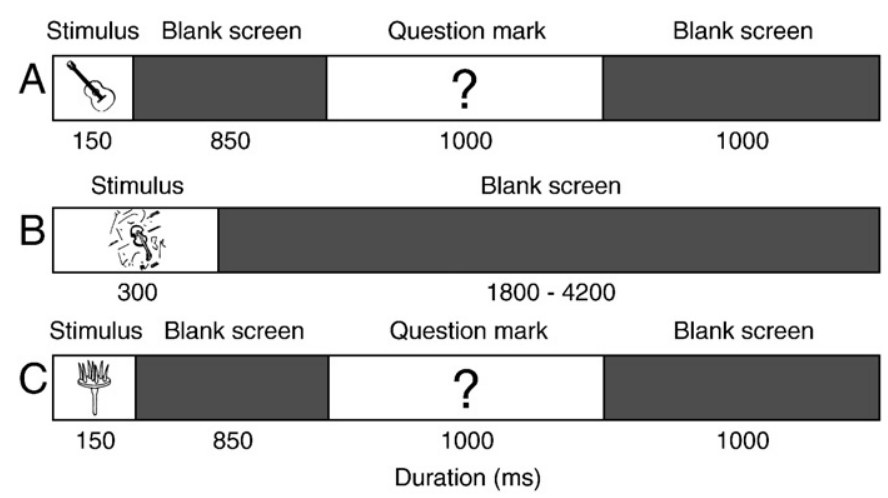

Fig. 1. Experimental design for (A) naming/categorization, (B) overt/covert object naming and $(C)$ naming of newly learned objects. 
experimental conditions: overt naming of the objects and their phonological categorization (detection of objects whose name started with a vowel; only the non-target trials were analyzed).

\section{Overt/Covert object naming}

Eleven healthy subjects ( 7 females, 4 males) were shown line drawings of everyday objects (100 images). The experiment was arranged as a block design, with alternating 30 -s task periods and 21$s$ rest periods. In a task period, 10 drawings of objects were shown for $300 \mathrm{~ms}$ with randomized intervals of $1.8-4.2 \mathrm{~s}$. There was no delay in naming, i.e., the subjects could start to name the object as soon as they saw an image (Fig. 1B). The present study focused on two of the original experimental conditions: overt and covert naming of object images.

\section{Naming/Categorization of newly learned objects}

Ten healthy subjects ( 5 females, 5 males) were taught the names of previously unknown pictured items $(\mathrm{N}=50)$. The MEG data examined in the present study was collected after the training phase. The stimuli were displayed for $150 \mathrm{~ms}$, followed by a blank screen for $850 \mathrm{~ms}$ (Fig. 1C). A question mark was then presented for $1 \mathrm{~s}$, prompting the subject to respond. The present study focused on two of the original experimental conditions: overt naming of the objects and their phonological categorization (detection of objects whose name started with the letter ' $r$ '; only the non-target trials were analyzed).

\section{Sensor-level analysis}

The signal level of phase-locked activity was quantified from evoked responses averaged across trials. The relevant frequency bands of rhythmic activity were identified from Morlet-wavelet based sensor-level time-frequency representations (TFR; TallonBaudry et al., 1997). Modulation of the signal level in those bands was quantified using temporal spectral evolution curves (TSE; Salmelin and Hari, 1994). For both response types, each recording site was represented by the vector sum of the two planar gradiometers at that location. Sensors above active brain areas were identified based on their maximum response amplitude: signals exceeding 6.1 times the standard deviation of the amplitude variation in the baseline interval ( $-200--100 \mathrm{~ms})$, corresponding to a p-level of $10^{-10}$, were considered significant. This conservative threshold was chosen in an attempt to enhance the spatial specificity of the sensor-level effects in the presence of the inherent field spread confound and to account for the large number of multiple comparisons (102 sensor locations, 1050 time points). The sensors thus identified as "active" (value 1, "non-active" 0 ) in individual subjects were then summed into a group-level consistency map of "active" sensors (number of subjects per recording site). Correspondence maps between evoked responses and modulation of rhythmic activity were generated for each individual subject by giving a sensor site the value 1 only when both response types were significant (otherwise, the sensor site assumed the value 0 ). The specific goal was to evaluate the global spatial correspondence between the two types of phenomena and, thus, areas showing suppression or enhancement of rhythmic activity were not distinguished from each other in the analysis. A group-level consistency map of evoked-rhythm correspondence was compiled by summing over subjects.

\section{Source-level analysis of rhythmic activity}

The frequency bands identified in the sensor-level analysis were analyzed at the source level using erDICS. The erDICS analysis followed the power mapping procedures described earlier (Laaksonen et al., 2008). In erDICS, the beamformer parameters are estimated for each time point using wavelet-based frequency decomposition.
Averaging across a short time window of 20 samples was employed to increase the signal-to-noise ratio of the covariance estimate. This operation reduced the time resolution to $33 \mathrm{~ms}$. The power level in the prestimulus baseline interval $(-200--100 \mathrm{~ms}$ with respect to stimulus onset) was subtracted from the power maps and salient positive and negative deflections were localized by identifying local max$\mathrm{ima} /$ minima from the power maps of each subject. Power maps were estimated over a time period of $0-3 \mathrm{~s}$ relative to the stimulus onset.

The center points of the local maxima/minima were identified from the individual power maps and stored. The time series of activation in these source locations were estimated with the DICS frequency-domain beamformer (Gross et al., 2001). Accepted sources had to meet two criteria: First, the time courses displayed modulation that exceeded twice the baseline standard deviation (corresponding to $\mathrm{P}<0.05$ ) contiguously for at least $250 \mathrm{~ms}$. Second, the final set of sources accounted for the modulations recorded by the entire MEG sensor array, explaining $>80 \%$ of the MEG data variance (analogously to the "goodness-of-fit" criterion used in the source analysis of evoked responses (Hämäläinen et al., 1993)). The modulation of rhythmic activity was quantified with TSE curves. An example of the individual source identification procedure is illustrated in Fig. 2. The source areas were visualized using the FreeSurfer software package (http://surfer.nmr.mgh.harvard.edu/). The source area center points were projected onto the inflated brain surface and then slightly enlarged for better visibility.

For group-level description of the individually determined sources of rhythmic activity, the identified areas were clustered by location, separately for the three experiments and the selected frequency bands. For each experiment, the two tasks (overt, covert) were pooled together. The source points were transformed into a common
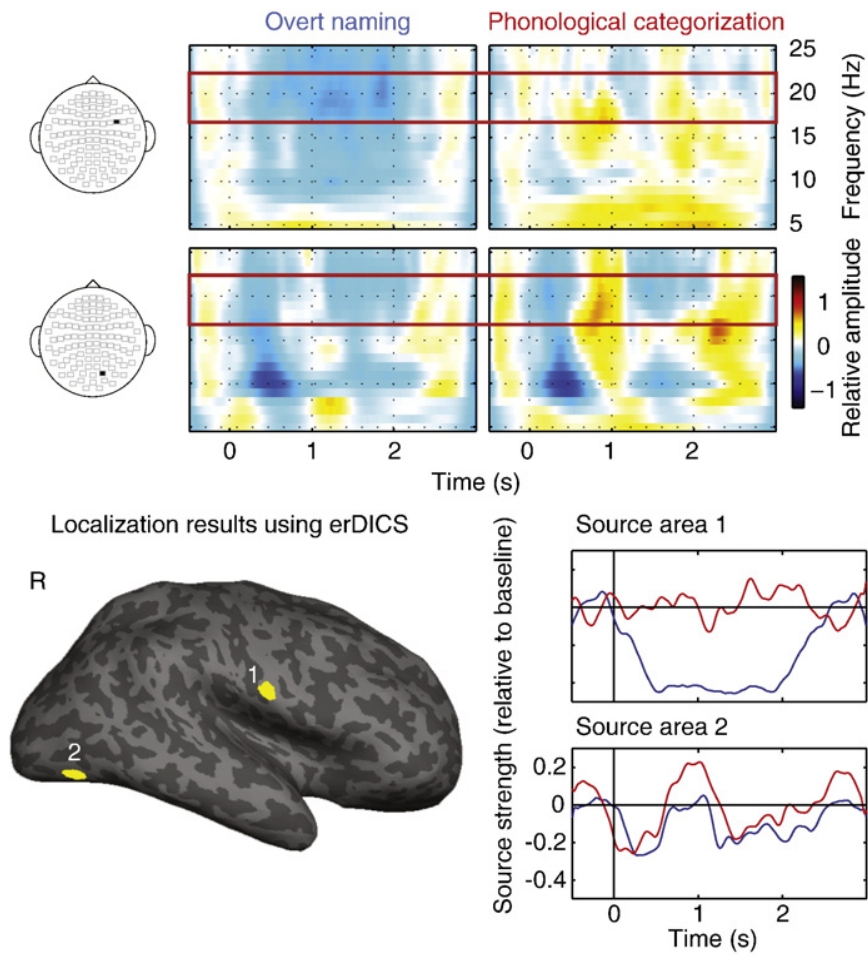

Fig. 2. Procedure for localizing sources of rhythmic activity in individual subjects using erDICS. MEG data from object naming/categorization experiment. Top: TFR plots show the power modulation as a function of time, with the selected frequency band marked by a red box. Bottom: The localized sources are visualized as yellow patches on an inflated brain surface of the right hemisphere. The power modulation as a function of time (TSE curves, low-pass filtered with corner frequency of $15 \mathrm{~Hz}$, expressed relative to the power level in the prestimulus baseline interval) in the two source areas was estimated with the DICS beamformer. 
brain coordinate system using an elastic transformation (Schormann and Zilles, 1998) between the MR images of each subject's brain and those of the common brain. The clustering was based on a predefined set of regions of interests, or labels, defined by an automatic parcellation procedure in FreeSurfer (Desikan et al., 2006; Fischl et al., 2004). A region was considered "active" when at least half of the subjects $(\geq 6)$ per experiment had a source belonging to that label.

\section{Source-level analysis of evoked responses}

The source-level analysis of the phase-locked evoked responses is described in detail in the original publications (Hultén et al., 2009; Liljeström et al., 2009; Vihla et al., 2006). The neural sources and their time courses of activation were determined by means of guided current modeling (Equivalent Current Dipole analysis, ECD; Hämäläinen et al., 1993). Independent spatiotemporal components were added until they explained a significant amount of MEG data variance $(>80 \%)$. Distributed source analysis (Minimum Norm Estimate, MNE; Hämäläinen and Ilmoniemi, 1994), additionally performed on one data set, confirmed the ECD result (Liljeström et al., 2009). Note that the source analysis of the overt naming data in the overt/covert object naming experiment was not reported in the original publication (Liljeström et al., 2009).

\section{Source-level comparison between rhythmic activity and evoked responses}

The source areas of the rhythmic activity and the ECD location parameters of the evoked responses were compared by calculating, for each source location, the minimum distance to any source area of the other type of activity; in the analysis, suppression or enhancement of rhythmic activity was not distinguished from each other. The sources were deemed to be from the same brain label, if the distance between them was less than $20 \mathrm{~mm}$. MEG cannot well distinguish sources with similar orientations of current flow that are closer than this (Liljeström et al., 2005). The estimation of distance was done separately for each subject, with the two tasks per experiment pooled together. "Hit rates" were calculated in two ways: (i) per label, with the rhythmic activity as the starting point, the percentage of subjects who had a source of evoked response in the same label with a rhythmic source, and (ii) per subject, with the evoked responses as the starting point, the percentage of sources of evoked responses with a source of rhythmic activity close-by (within $20 \mathrm{~mm}$ ). This fairly lenient distance criterion was adopted in order to accommodate for possible inaccuracies in localization of the source areas. ECDs were pooled together from the two tasks per experiment (the object naming/categorization experiment used the same set of ECDs for both tasks).

Comparison of temporal and functional differences between the two measures of activity was limited to spatially highly similar source areas of rhythms and evoked responses, i.e., labels where at least 5 subjects had both an evoked and a rhythmic source in both the overt and covert variant of a task. In these labels, the time courses of the task effects of the two types of measures were compared. For time windows with statistically significant task effects, we additionally estimated the single-trial correlation between amplitude levels of rhythmic activity and evoked responses.

\section{Results}

\section{Sensor-level analysis}

Evoked responses showed a wide spatial spread across the sensor array, consistently across subjects (Fig. 3A). Modulation of 10- and $20-\mathrm{Hz}$ rhythms displayed slightly more spatial specificity, with consistent $10-\mathrm{Hz}$ effects on many posterior sensors but less over frontal regions (Fig. 3B) and $20-\mathrm{Hz}$ effects over the occipito-parietal region and sensorimotor areas, especially on the left side (Fig. 3C). The most consistent overlap between evoked responses and rhythmic activity (Figs. 3D-E) occurred on sensors over the occipital (10-Hz vs. evoked) and occipito-parietal cortex (both 10- and 20-Hz vs. evoked) and on sensors covering the sensorimotor cortex (especially $20-\mathrm{Hz}$ vs. evoked, on the left). As the sensor-level view is spatially quite blurred, and the same sensor may pick up activity from multiple separate source areas, source-level analysis is necessary for a detailed, quantitative evaluation.

\section{Cortical sources of rhythmic activity}

Based on strong task-related modulations of rhythmic activity in the TFR plots (cf. Fig. 2), the source-level analysis was focused on two frequency bands: 7-12 and 17-22 Hz. Fig. 4 shows the localization results of rhythmic activity using the individual-level approach and subsequent clustering to the predefined cortical labels. Across the three data sets, modulation of rhythmic activity was most consistently detected in the visual and motor cortex, and also in the parietal and superior temporal regions. For comparison, a direct group-level mapping of power modulation in these frequency ranges showed an overall comparable but spatially more widely spread pattern (Supplementary Information and Supplementary Fig. 1).
A

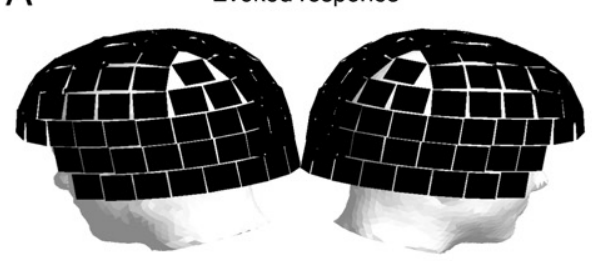

B
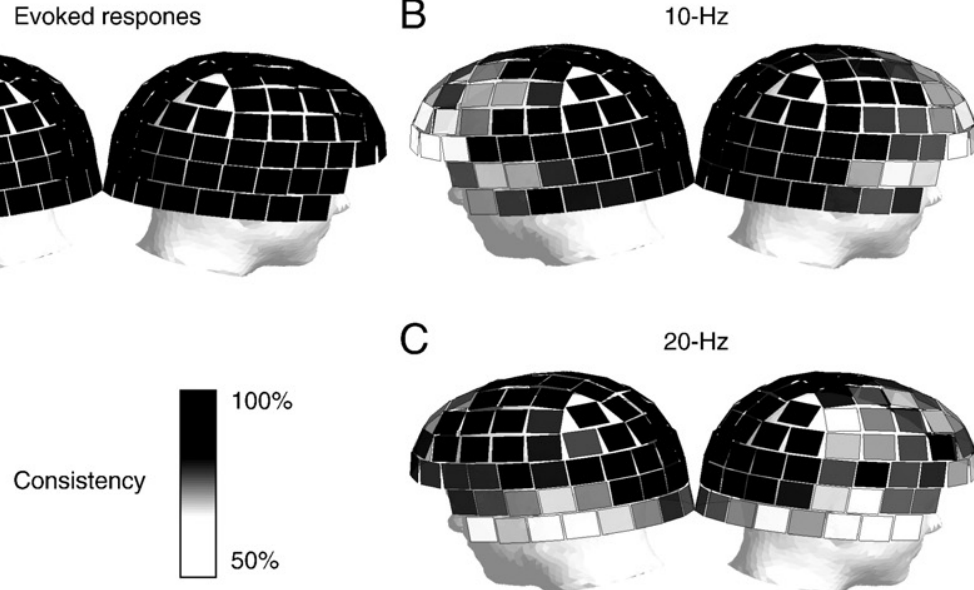

C

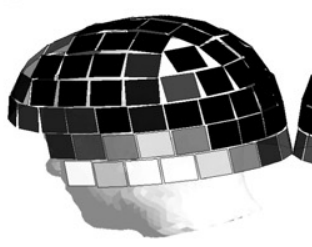

$20-\mathrm{Hz}$

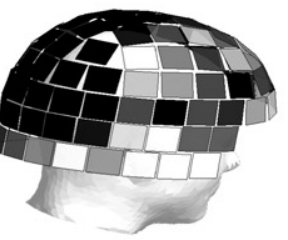

D $\quad 10-\mathrm{Hz}$ vs. evoked

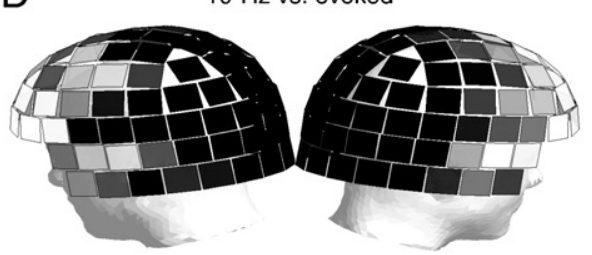

E

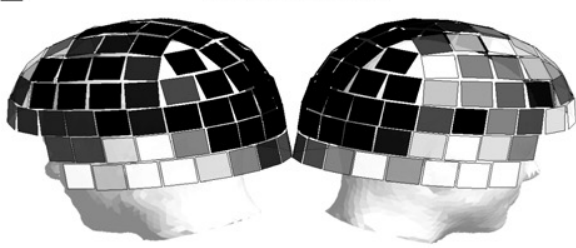

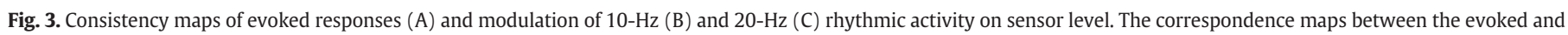
rhythmic responses (D-E) were first evaluated for individual subjects and then merged across subjects and data sets. 

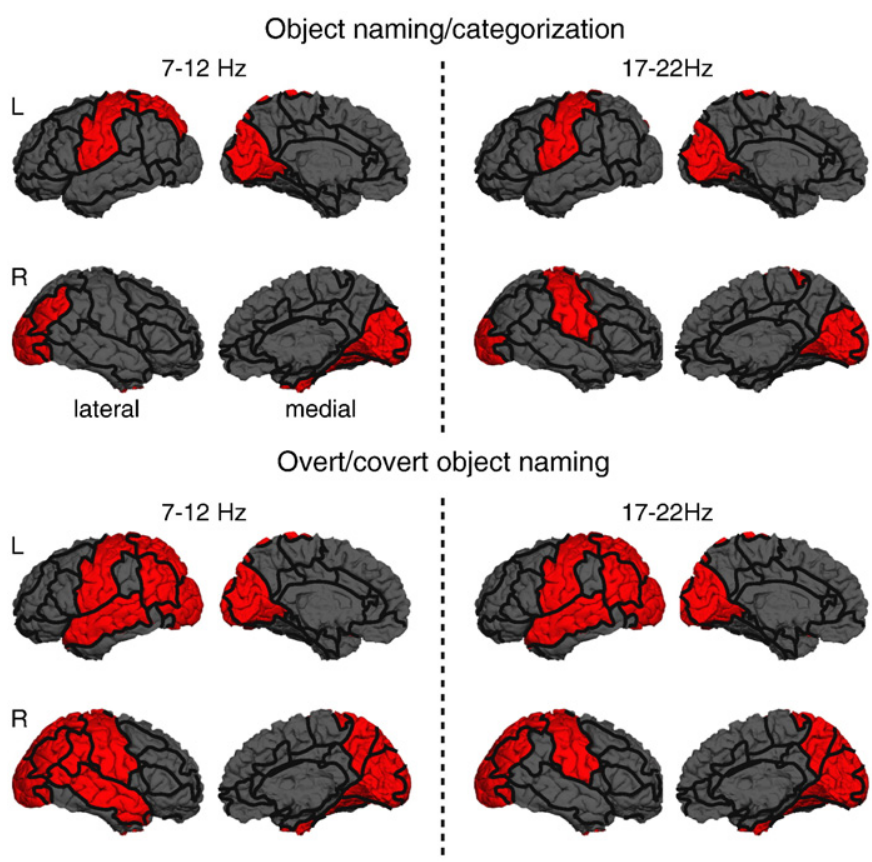

Naming/categorization of newly learned objects

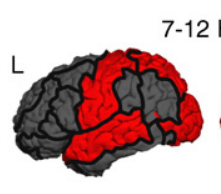

$-12 \mathrm{~Hz}$
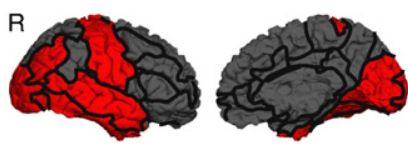

$17-22 \mathrm{~Hz}$

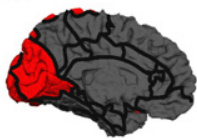

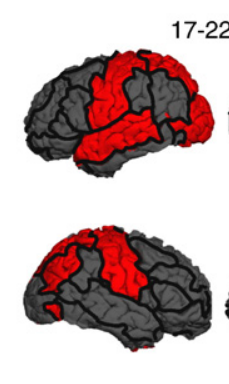

!

Fig. 4. Source areas of rhythmic activity localized using erDICS and visualized on brain surfaces (lateral and medial views of the left and right hemispheres). Red labels indicate areas in which modulation of rhythmic activity in each experiment (three data sets, from top to bottom) and in the specified frequency range (left: 7-12 Hz, right: $17-22 \mathrm{~Hz}$ ) was identified in at least half of the subjects.

To complement power mapping at the prominent $10-\mathrm{Hz}$ and $20-$ $\mathrm{Hz}$ frequency bands, we additionally estimated the spatial distribution of power in the gamma range, with focus on the so called "high gamma" at $60-100 \mathrm{~Hz}$ (Supplementary Information and Supplementary Fig. 2). In the individual mapping, activity was found in the temporal, frontal and visual cortex, variably across subjects. Significant group-level activity was primarily concentrated to the visual cortex. However, a large part of the gamma modulation seemed to reflect artifactual muscle activity from the mouth and the eyes (cf. Supplementary Figs. 2B-C). The gamma band results were not compared with the evoked responses.

\section{Cortical sources of evoked activity}

Overall, 5-11 separable source areas (mean 8.6, standard deviation 1.5) were identified across the subjects and tasks in the three experiments (Hultén et al., 2009; Liljeström et al., 2009; Vihla et al., 2006). ECD analysis of object naming showed, similarly in all three studies, an initial transient response in the visual cortex $(<200 \mathrm{~ms})$ that was followed by more sustained occipital activation and a salient response in the parietal cortex. After $\sim 300 \mathrm{~ms}$ the activation spread to the temporal and frontal cortex.
Cortical correspondence of rhythmic activity and evoked responses

The group-level convergence between rhythmic activity and evoked responses in the various brain regions is presented in Table 1 . Across the three experiments (each with two conditions), two frequency ranges and 29 defined regions of interest in both hemispheres, thus altogether 348 examined labels, 61 were marked active (sources of rhythmic modulation in at least 6 subjects, Fig. 4). Out of these labels, 22 exceeded the hit rate of 50\%, with the lateral aspect of the right occipital cortex the most consistent convergence area across experiments. Other areas of high convergence were the medial occipital and the left pericentral cortex. When comparing the likelihood of finding sources of rhythmic activity close to sources of evoked responses per subject, the hit rate was on average $30 \%$, exceeding $50 \%$ only in 4 cases out of 31 (Table 2 ).

The temporal characteristics were inspected in labels that consistently showed both rhythmic and evoked activity in both the overt and covert variant of a task. Only 9 labels out of the 19 with better than $50 \%$ convergence of rhythmic and evoked activity contained a large enough absolute number of subjects to facilitate comparison, i.e., at least 5 subjects with both types of sources in both the overt and covert task. The overt vs. covert nature of the tasks influenced the spatial distribution of the sources of the evoked responses, which also served to reduce the eventual number of converging labels. Fig. 5 exemplifies the relationship between evoked responses and modulation of rhythmic activity through time courses of activation in the right lateral occipital and left pericentral (sensorimotor) cortex in the object naming/categorization experiment, in the $10-\mathrm{Hz}$ range. In the occipital area, no statistically significant task effect was observed in the rhythmic modulation, but the evoked responses showed an early task effect at around 100-200 ms. Statistically significant task differences emerged in the pericentral area, with stronger suppression of $10-\mathrm{Hz}$ rhythmic activity in overt naming than silent categorization; a stronger phase-locked evoked response for overt naming approached significance. Notably, the rhythmic activity differentiated between the tasks in both an earlier (500-700 ms) and later time window (1600-2500 ms) whereas the evoked response indicated a trend for task effects only in the later time window (1200-2000 ms), when the subject gave an overt response. In single-trial analysis, no significant correlation between rhythmic activity and evoked responses was detected during the task-effect time windows of either measure.

\section{Discussion}

We used three MEG data sets from experiments that addressed high-level cognitive processing to investigate the spatial correspondence between evoked responses and event-related modulation of rhythmic activity. The activation patterns revealed by evoked responses have been analyzed and reported previously (Hultén et al., 2009; Liljeström et al., 2009; Vihla et al., 2006). The present study inspected the brain oscillations in two frequency bands $(\sim 10 \mathrm{~Hz}$, $\sim 20 \mathrm{~Hz}$ ) that displayed prominent modulation of rhythmic activity in these data sets. Modulations in these frequency bands are the most typical oscillatory effects in MEG measurements (Hari and Salmelin, 1997).

At the sensor level, evoked responses and rhythmic activity coincided primarily on sensors located over posterior brain regions and sensorimotor cortex. This simple comparison was followed by our best effort in localizing both evoked and rhythmic source areas. The ideal comparison between the two markers of neural activity would be with the same operator into the same source space. However, there are some practical problems against this. Beamformers do not work well with evoked responses (Van Veen et al., 1997) and distributed source models, such as MCE (Jensen and Vanni, 2002; Uutela et al., 1999), have poorer localization accuracy for rhythms than do 
Table 1

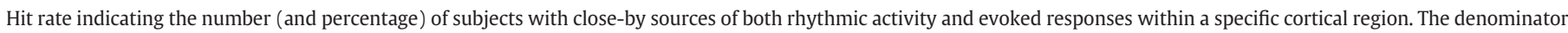
gives the total number of subjects with a source of rhythmic activity in that region.

\begin{tabular}{|c|c|c|c|c|c|c|}
\hline \multirow[t]{2}{*}{ Cortical area } & \multirow{2}{*}{$\begin{array}{l}\begin{array}{l}\text { Object naming/ } \\
\text { categorization }\end{array} \\
7-12 \mathrm{~Hz}\end{array}$} & \multirow[t]{2}{*}{$\begin{array}{l}\text { Overt/Covert } \\
\text { object naming }\end{array}$} & \multirow[t]{2}{*}{$\begin{array}{l}\text { Naming of } \\
\text { learned objects }\end{array}$} & $\begin{array}{l}\text { Object naming/ } \\
\text { categorization }\end{array}$ & \multirow[t]{2}{*}{$\begin{array}{l}\text { Overt/Covert } \\
\text { object naming }\end{array}$} & \multirow[t]{2}{*}{$\begin{array}{l}\text { Naming of } \\
\text { learned objects }\end{array}$} \\
\hline & & & & $17-22 \mathrm{~Hz}$ & & \\
\hline \multicolumn{7}{|l|}{ Right hemisphere } \\
\hline Medial occipital & $3 / 7(43)$ & $7 / 8(88)$ & $3 / 6(50)$ & $7 / 10(70)$ & $4 / 8(50)$ & $3 / 6(50)$ \\
\hline Lateral occipital & $8 / 8(100)$ & $8 / 9(89)$ & $5 / 8(63)$ & $7 / 9(78)$ & $4 / 10(40)$ & \\
\hline Fusiform & $4 / 7(57)$ & $5 / 8(63)$ & $3 / 6(50)$ & & $3 / 10(30)$ & $1 / 6(17)$ \\
\hline Superior parietal & & $2 / 9(22)$ & & & $3 / 8(38)$ & $4 / 6(66)$ \\
\hline Inferior parietal & $2 / 7(29)$ & $4 / 9(44)$ & 4/7 (57) & & $4 / 10(40)$ & $3 / 6(50)$ \\
\hline Precuneus & & $2 / 8(25)$ & & & $2 / 6(33)$ & \\
\hline Supramarginal & & $4 / 6(67)$ & & & & \\
\hline Superior temporal & & $2 / 8(25)$ & $1 / 7(14)$ & & & \\
\hline Inferior temporal & & & $4 / 6(67)$ & & & \\
\hline Pericentral & & $3 / 8(38)$ & $3 / 6(50)$ & $3 / 6(50)$ & $4 / 9(44)$ & $2 / 9(22)$ \\
\hline \multicolumn{7}{|l|}{ Left hemisphere } \\
\hline Medial occipital & $4 / 8(50)$ & $5 / 8(63)$ & $6 / 10(60)$ & 2/7 (29) & $5 / 9(56)$ & $4 / 9(44)$ \\
\hline Lateral occipital & & $5 / 9(56)$ & $4 / 8(50)$ & & $4 / 7(57)$ & $3 / 7(43)$ \\
\hline Superior parietal & $4 / 6(67)$ & $4 / 9(44)$ & $2 / 6(33)$ & & $3 / 10(30)$ & $1 / 6(17)$ \\
\hline Inferior parietal & & $2 / 7$ (29) & & & & \\
\hline Superior temporal & & $1 / 6(17)$ & 2/7 (29) & & $3 / 6(50)$ & 4/6 (67) \\
\hline Pericentral & $5 / 7(71)$ & $3 / 7(43)$ & $4 / 7(57)$ & $4 / 8(50)$ & $4 / 10(40)$ & $5 / 7(71)$ \\
\hline
\end{tabular}

beamformers (Liljeström et al., 2005). Our choice was, thus, to use for each response type methods which have been proven to work the best. We further sought to alleviate possible confounds or biases by using a very lenient distance criterion $(20 \mathrm{~mm})$ when looking for overlap in source areas. This loose criterion should allow us to find correspondences between evoked and rhythmic source areas, when such exist.

The hit rate measures indicated that the overlap between sources of rhythmic activity and evoked responses was quite small, with the right lateral occipital and left pericentral cortices the most consistent convergence areas across experiments. This result is in line with the crude sensor-level analysis. It thus seems that evoked responses and cortical rhythms provided mostly complementary information about neural processing in these high-level cognitive tasks. Even in the few areas with spatial overlap between evoked responses and cortical rhythms, their time behaviors and functional differentiation could be markedly different. Based on the spatiotemporal properties of the two measures, it seems that they reflect different brain processes, or at least that their link is not straightforward. The underlying neural activities might differ in the way they reflect information processing or by the time scale they transfer/integrate information as evoked activity is typically observed for shorter durations than rhythmic modulations. Evoked responses constitute the part of neural activity that

Table 2

Hit rate indicating, per subject, the number (and percentage) of sources of evoked responses with a source of rhythmic activity in close vicinity. The denominator is the total number of ECDs pooled from the two tasks per experiment (the object naming/ categorization experiment used the same set of ECDs for both tasks). Each experiment included 10-11 subjects (S01-S11).

\begin{tabular}{llll}
\hline Subject & $\begin{array}{l}\text { Object naming/ } \\
\text { categorization }\end{array}$ & $\begin{array}{l}\text { Overt/Covert } \\
\text { object naming }\end{array}$ & $\begin{array}{l}\text { Naming/Categorization } \\
\text { of newly learned objects }\end{array}$ \\
\hline S01 & $3 / 10(30)$ & $10 / 17(58)$ & $5 / 14(36)$ \\
S02 & $0 / 9(0)$ & $4 / 16(25)$ & $4 / 15(27)$ \\
S03 & $4 / 11(36)$ & $5 / 16(31)$ & $2 / 15(13)$ \\
S04 & $3 / 10(30)$ & $10 / 21(48)$ & $2 / 18(11)$ \\
S05 & $3 / 10(30)$ & $6 / 20(30)$ & $5 / 17(29)$ \\
S06 & $2 / 10(20)$ & $10 / 14(71)$ & $1 / 12(8)$ \\
S07 & $2 / 11(18)$ & $8 / 17(47)$ & $4 / 15(27)$ \\
S08 & $3 / 10(30)$ & $0 / 18(0)$ & $6 / 14(42)$ \\
S09 & $2 / 10(20)$ & $6 / 15(40)$ & $7 / 16(44)$ \\
S10 & $2 / 10(20)$ & $6 / 15(40)$ & $9 / 17(53)$ \\
S11 & & $8 / 12(67)$ & \\
\hline
\end{tabular}

occurs with remarkably similar timing from trial to trial, thus probably representing a functionally strongly predetermined sequence of activation, possibly with a fair bottom-up emphasis.

For one data set, the gamma band $(60-100 \mathrm{~Hz})$ was additionally investigated. In this frequency band, notable activity was limited to the visual cortex. This source locus is in agreement with MEG studies that have investigated gamma activity and also localized its cortical source areas (Hoogenboom et al., 2006; Muthukumaraswamy et al., 2010). Importantly, though, we also observed that, in non-invasive electromagnetic recordings (and, presumably, in EEG), muscle movement from the mouth area and the eyes can generate a gammafrequency electromagnetic field that, when source localization procedures limit the solution space to the cortex, may be erroneously interpreted as stimulus- or task-related neural activity. Based on these findings, we would urge caution when interpreting gamma
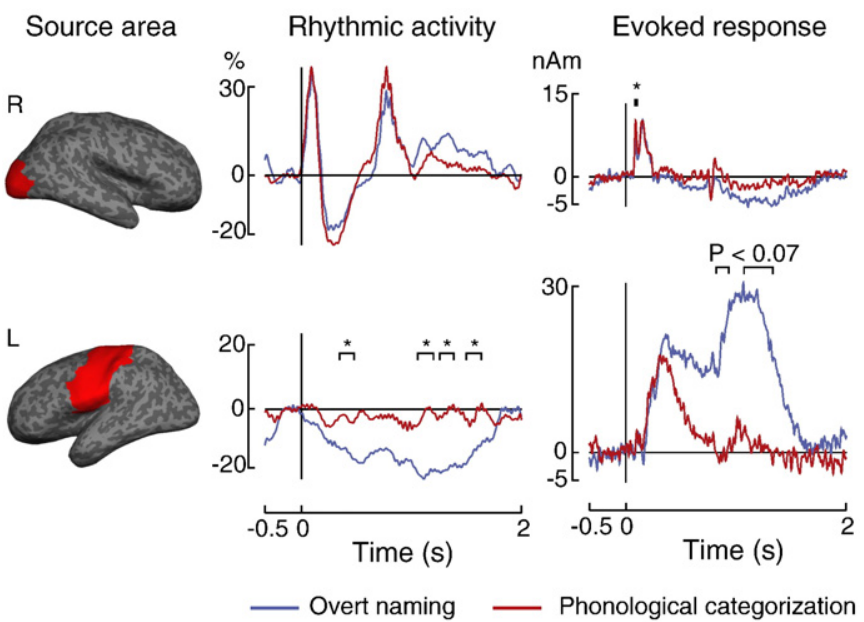

Fig. 5. Comparison of the time courses of evoked responses and induced modulation of rhythmic activity. Evoked responses and TSE curves of 10-Hz activity (low-pass filtered with corner frequency of $15 \mathrm{~Hz}$ ) are plotted for two brain regions where both types of neural activity were detected in at least 5 subjects; TSE modulation strength is expressed relative to the power level in the prestimulus baseline interval and evoked response amplitude is in nAm. Data from the object naming/categorization experiment The asterisks indicate significant differences between the two tasks (paired permutation test, $\left.{ }^{*} \mathrm{P}<0.05\right)$. In the left sensorimotor cortex, the apparent difference in the evoked responses approached significance $(\mathrm{P}<0.07)$ in the late time window. 
findings near orbitofrontal, inferior frontal or anterior temporal areas (possible eye movement and/or mouth movement artifacts).

The role of longer lasting rhythmic responses is speculated to range from idling (Pfurtscheller et al., 1996a, 1996b) to retaining information about the sequence of events available over several hundreds of milliseconds, thus facilitating integration of information from sequential events (Dinse et al., 1997). Alpha band rhythms have been linked to active processing (Palva and Palva, 2007) and they seem to play a role in visual attention guidance, either actively (Van Der Werf et al., 2008) or in "pulses" (Mathewson et al., 2009). Beta band rhythms have been linked to upkeeping of expectations or status quo (Engel and Fries, 2010). Furthermore, neuronal oscillations have been suggested to be instrumental in setting up neuronal synchrony over large distances as oscillating networks can achieve zero-lag synchronization despite considerable conduction delays, via intermediate oscillators (Singer, 1993). The phase synchronization between remote neuronal assemblies, in turn, has been proposed to promote efficient information transfer and integration of information, thus linking together different brain regions into functional networks (Fries, 2005; Varela et al., 2001).

One hypothesis for the link between rhythms and evoked responses is that the phase of rhythmic activity forms the evoked response (Makeig et al., 2002). In this study, we only focused on salient power modulations of rhythmic activity and cannot, therefore, make statements on the phase resetting. Even if this were a valid explanation of evoked responses in areas where both rhythmic activity and evoked responses coexist, it would still leave open the question of how power modulations of brain oscillations relate to phase resetting. Another proposed mechanism for generation of phase-locked event-related responses is the asymmetric amplitude modulation of brain oscillations (Mazaheri and Jensen, 2008; Nikulin et al., 2007). At a close perusal of the MEG signal, we observed a considerable interindividual and intertrial variation of cortical oscillations. Robust hypothesis testing of the different proposed models will, thus, probably require far larger subject populations (on the order of hundreds) than are typically used in imaging experiments (10-15 subjects).

Modulation of rhythmic activity was detected in areas that show strong rhythmicity at rest, such as the occipital visual areas and the parieto-occipital sulcus. Task performance additionally revealed rhythmicity in central sulcus in the proximity of the face/mouth representation areas, in agreement with prior studies on verbal and nonverbal mouth movements (Saarinen et al., 2006; Salmelin et al., 1995; Salmelin et al., 2000). Nevertheless, evoked responses covered cortical areas that showed no salient rhythmicity either at rest or in task performance and, vice versa, rhythmic modulation was detected in areas that did not manifest phase-locked responses.

To summarize, the weak spatio-temporo-functional overlap between the evoked responses and modulation of rhythmic activity in our cognitive tasks, with altogether 31 recordings, suggests that the phase-locked evoked responses and rhythmic activity are largely disconnected and that a holistic view via both measures would be valuable for an accurate portrayal of brain activity.

Supplementary materials related to this article can be found online at doi:10.1016/j.neuroimage.2011.11.087.

\section{Acknowledgments}

We thank Minna Vihla for her contributions in the object naming/ categorization experiment, Antti Jalava for assistance with the FreeSurfer software and Mika Seppä for help with elastic transforms. This work was supported by the Academy of Finland (Centre of Excellence Programme 2006-2011, personal grant to R.S.), Sigrid Jusélius Foundation, Finnish Foundation for Technology Promotion, Emil Aaltonen Foundation and Finnish Funding Agency for Technology and Innovation (SalWe Strategic Center for Science, Technology and Innovation in Health and Well-being).

\section{References}

Brenner, D., Williamson, S.J., Kaufman, L., 1975. Visually evoked magnetic fields of the human brain. Science 190, 480-482.

Brenner, D., Lipton, J., Kaufman, L., Williamson, S.J., 1978. Somatically evoked magnetic fields of the human brain. Science 199, 81-83.

Desikan, R.S., Segonne, F., Fischl, B., Quinn, B.T., Dickerson, B.C., Blacker, D., Buckner, R.L., Dale, A.M., Maguire, R.P., Hyman, B.T., Albert, M.S., Killiany, R.J., 2006. An automated labeling system for subdividing the human cerebral cortex on MRI scans into gyral based regions of interest. Neurolmage 31, 968-980.

Dinse, H.R., Kruger, K., Akhavan, A.C., Spengler, F., Schoner, G., Schreiner, C.E., 1997. Low-frequency oscillations of visual, auditory and somatosensory cortical neurons evoked by sensory stimulation. Int. J. Psychophysiol. 26, 205-227.

Engel, A.K., Fries, P., 2010. Beta-band oscillations-signalling the status quo? Curr. Opin. Neurobiol. 20, 156-165.

Fischl, B., van der Kouwe, A., Destrieux, C., Halgren, E., Segonne, F., Salat, D.H., Busa, E., Seidman, L.J., Goldstein, J., Kennedy, D., Caviness, V., Makris, N., Rosen, B., Dale, A.M., 2004. Automatically parcellating the human cerebral cortex. Cereb. Cortex $14,11-22$

Fries, P., 2005. A mechanism for cognitive dynamics: neuronal communication through neuronal coherence. Trends Cogn. Sci. 9, 474-480.

Gross, J., Kujala, J., Hämäläinen, M., Timmermann, L., Schnitzler, A., Salmelin, R., 2001. Dynamic imaging of coherent sources: studying neural interactions in the human brain. Proc. Natl. Acad. Sci. U. S. A. 98, 694-699.

Hämäläinen, M.S., Ilmoniemi, R.J., 1994. Interpreting magnetic fields of the brain: minimum norm estimates. Med. Biol. Eng. Comput. 32, 35-42.

Hämäläinen, M., Hari, R., Ilmoniemi, R.J., Knuutila, J., Lounasmaa, O.V., 1993. Magnetoencephalography - theory, instrumentation, and applications to noninvasive studies of the working human brain. Rev. Mod. Phys. 65, 413-497.

Hari, R., Salmelin, R., 1997. Human cortical oscillations: a neuromagnetic view through the skull. Trends Neurosci. 20, 44-49.

Hari, R., Aittoniemi, K., Järvinen, M.L., Katila, T., Varpula, T., 1980. Auditory evoked transient and sustained magnetic fields of the human brain. Localization of neural generators. Exp. Brain Res. 40, 237-240.

Helenius, P., Salmelin, R., Service, E., Connolly, J.F., 1998. Distinct time courses of word and context comprehension in the left temporal cortex. Brain 121, 1133-1142.

Hoogenboom, N., Schoffelen, J.M., Oostenveld, R., Parkes, L.M., Fries, P., 2006. Localizing human visual gamma-band activity in frequency, time and space. NeuroImage 29, 764-773.

Hultén, A., Vihla, M., Laine, M., Salmelin, R., 2009. Accessing newly learned names and meanings in the native language. Hum. Brain Mapp. 30, 976-989.

Indefrey, P., Levelt, W.J., 2004. The spatial and temporal signatures of word production components. Cognition 92, 101-144.

Ishii, R., Shinosaki, K., Ukai, S., Inouye, T., Ishihara, T., Yoshimine, T., Hirabuki, N., Asada, H., Kihara, T., Robinson, S.E., Takeda, M., 1999. Medial prefrontal cortex generates frontal midline theta rhythm. Neuroreport 10, 675-679.

Jensen, O., Tesche, C.D., 2002. Frontal theta activity in humans increases with memory load in a working memory task. Eur. J. Neurosci. 15, 1395-1399.

Jensen, O., Vanni, S., 2002. A new method to identify multiple sources of oscillatory activity from magnetoencephalographic data. Neurolmage 15, 568-574.

Jensen, O., Gelfand, J., Kounios, J., Lisman, J.E., 2002. Oscillations in the alpha band $(9-12 \mathrm{~Hz})$ increase with memory load during retention in a short-term memory task. Cereb. Cortex 12, 877-882.

Jerbi, K., Ossandon, T., Hamame, C.M., Senova, S., Dalal, S.S., Jung J., Minotti, L., Bertrand, O., Berthoz, A., Kahane, P., Lachaux, J.P., 2009. Task-related gamma-band dynamics from an intracerebral perspective: review and implications for surface EEG and MEG. Hum. Brain Mapp. 30, 1758-1771.

Karhu, J., Hari, R., Lu, S.T., Paetau, R, Rif, J., 1991. Cerebral magnetic fields to lingual stimulation. Electroencephalogr. Clin. Neurophysiol. 80, 459-468.

Laaksonen, H., Kujala, J., Salmelin, R., 2008. A method for spatiotemporal mapping of event-related modulation of cortical rhythmic activity. NeuroImage 42, 207-217.

Liljeström, M., Kujala, J., Jensen, O., Salmelin, R., 2005. Neuromagnetic localization of rhythmic activity in the human brain: a comparison of three methods. Neurolmage 25, 734-745.

Liljeström, M., Hultén, A., Parkkonen, L., Salmelin, R., 2009. Comparing MEG and fMRI views to naming actions and objects. Hum. Brain Mapp. 30, 1845-1856.

Makeig, S., Westerfield, M., Jung, T.P., Enghoff, S., Townsend, J., Courchesne, E., Sejnowski, T.J., 2002. Dynamic brain sources of visual evoked responses. Science 295, 690-694.

Mäkinen, V., Tiitinen, H., May, P., 2005. Auditory event-related responses are generated independently of ongoing brain activity. NeuroImage 24, 961-968.

Mathewson, K.E., Gratton, G., Fabiani, M., Beck, D.M., Ro, T., 2009. To see or not to see: prestimulus alpha phase predicts visual awareness. J. Neurosci. 29, 2725-2732.

Mazaheri, A., Jensen, O., 2006. Posterior alpha activity is not phase-reset by visual stimuli. Proc. Natl. Acad. Sci. U. S. A. 103, 2948-2952.

Mazaheri, A., Jensen, O., 2008. Asymmetric amplitude modulations of brain oscillations generate slow evoked responses. J. Neurosci. 28, 7781-7787.

Muthukumaraswamy, S.D., Singh, K.D., Swettenham, J.B., Jones, D.K., 2010. Visual gamma oscillations and evoked responses: variability, repeatability and structural MRI correlates. NeuroImage 49, 3349-3357.

Nikulin, V.V., Linkenkaer-Hansen, K., Nolte, G., Lemm, S., Muller, K.R., Ilmoniemi, R.J., Curio, G., 2007. A novel mechanism for evoked responses in the human brain. Eur. J. Neurosci. 25, 3146-3154

Onton, J., Delorme, A., Makeig, S., 2005. Frontal midline EEG dynamics during working memory. Neurolmage 27, 341-356.

Palva, S., Palva, J.M., 2007. New vistas for alpha-frequency band oscillations. Trends Neurosci. $30,150-158$ 
Penny, W.D., Kiebel, S.J., Kilner, J.M., Rugg, M.D., 2002. Event-related brain dynamics. Trends Neurosci. 25, 387-389.

Pfurtscheller, G., Aranibar, A., 1977. Event-related cortical desynchronization detected by power measurements of scalp EEG. Electroencephalogr. Clin. Neurophysiol. 42, 817-826.

Pfurtscheller, G., Stancak Jr., A., Neuper, C., 1996a. Event-related synchronization (ERS) in the alpha band-an electrophysiological correlate of cortical idling: a review. Int. J. Psychophysiol. 24, 39-46.

Pfurtscheller, G., Stancak Jr., A., Neuper, C., 1996b. Post-movement beta synchronization. A correlate of an idling motor area? Electroencephalogr. Clin. Neurophysiol. 98, 281-293.

Saarinen, T., Laaksonen, H., Parviainen, T., Salmelin, R., 2006. Motor cortex dynamics in visuomotor production of speech and non-speech mouth movements. Cereb. Cortex 16, 212-222.

Salmelin, R., 2007. Clinical neurophysiology of language: the MEG approach. Clin. Neurophysiol. 118, 237-254.

Salmelin, R., Hari, R., 1994. Spatiotemporal characteristics of sensorimotor neuromagnetic rhythms related to thumb movement. Neuroscience 60, 537-550.

Salmelin, R., Hari, R., Lounasmaa, O.V., Sams, M., 1994. Dynamics of brain activation during picture naming. Nature 368, 463-465.

Salmelin, R., Hämäläinen, M., Kajola, M., Hari, R., 1995. Functional segregation of movement-related rhythmic activity in the human brain. Neurolmage 2, 237-243.

Salmelin, R., Schnitzler, A., Schmitz, F., Freund, H.J., 2000. Single word reading in developmental stutterers and fluent speakers. Brain 123, 1184-1202.

Schnitzler, A., Salenius, S., Salmelin, R., Jousmäki, V., Hari, R., 1997. Involvement of primary motor cortex in motor imagery: a neuromagnetic study. Neurolmage 6, 201-208.

Schormann, T., Zilles, K., 1998. Three-dimensional linear and nonlinear transformations: an integration of light microscopical and MRI data. Hum. Brain Mapp. 6, 339-347.
Shah, A.S., Bressler, S.L., Knuth, K.H., Ding, M., Mehta, A.D., Ulbert, I., Schroeder, C.E. 2004. Neural dynamics and the fundamental mechanisms of event-related brain potentials. Cereb. Cortex 14, 476-483.

Singer, W., 1993. Synchronization of cortical activity and its putative role in information processing and learning. Annu. Rev. Physiol. 55, 349-374.

Tallon-Baudry, C., Bertrand, O., Delpuech, C., Permier, J., 1997. Oscillatory gamma-band (30-70 Hz) activity induced by a visual search task in humans. J. Neurosci. 17, 722-734.

Taulu, S., Kajola, M., Simola, J., 2004. Suppression of interference and artifacts by the signal space separation method. Brain Topogr. 16, 269-275.

Tuladhar, A.M., ter Huurne, N., Schoffelen, J.M., Maris, E., Oostenveld, R., Jensen, O., 2007. Parieto-occipital sources account for the increase in alpha activity with working memory load. Hum. Brain Mapp. 28, 785-792.

Uutela, K., Hamalainen, M., Somersalo, E., 1999. Visualization of magnetoencephalographic data using minimum current estimates. Neurolmage 10, 173-180.

Van Der Werf, J., Jensen, O., Fries, P., Medendorp, W.P., 2008. Gamma-band activity in human posterior parietal cortex encodes the motor goal during delayed prosaccades and antisaccades. J. Neurosci. 28, 8397-8405.

Van Veen, B.D., van Drongelen, W., Yuchtman, M., Suzuki, A., 1997. Localization of brain electrical activity via linearly constrained minimum variance spatial filtering. IEEE Trans. Biomed. Eng. 44, 867-880.

Vanni, S., Revonsuo, A., Saarinen, J., Hari, R., 1996. Visual awareness of objects correlates with activity of right occipital cortex. Neuroreport 8, 183-186.

Vanni, S., Revonsuo, A., Hari, R., 1997. Modulation of the parieto-occipital alpha rhythm during object detection. J. Neurosci. 17, 7141-7147.

Varela, F., Lachaux, J.P., Rodriguez, E., Martinerie, J., 2001. The brainweb: phase synchronization and large-scale integration. Nat. Rev. Neurosci. 2, 229-239.

Vihla, M., Laine, M., Salmelin, R., 2006. Cortical dynamics of visual/semantic vs. phonological analysis in picture confrontation. NeuroImage 33, 732-738. 